\title{
A plea for useless curiosity
}

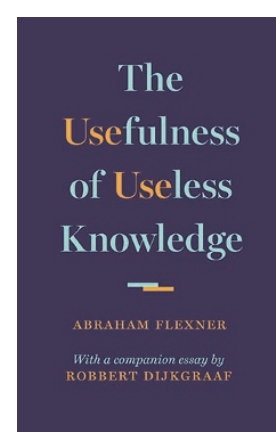

\section{The Usefulness of} Useless Knowledge

By Abraham Flexner and Robbert Dijkgraaf

PRINCETON UNIVERSITY

PRESS: 2017.

104 PP. $€ 7.50$

\footnotetext{
$\mathrm{T}$
} oday, the vast amount of available information constantly forces us into choosing between the important and the unimportant, the useful and the useless. In many contexts, making this distinction can be well justified, and we have been exceptionally successful in developing strategies and even algorithms for just this very task. How convenient it would be if we could apply these very same methods to address the problems of restricted research funding and the exponential growth of the number of scientific publications. However, scientific research is a tricky business it's an enterprise that often rails against a conclusive, immediate assessment of its success or necessary utility, by its very own intrinsic nature.
Abraham Flexner, the founding director of the Princeton Institute for Advanced Study, beautifully articulated the reasons for this in 1939. In a short essay, entitled The Usefulness of Useless Knowledge, Flexner convincingly argues that the path to those great discoveries, which ultimately turn out to be beneficial to mankind, is intrinsically rooted in curiosity, rather than an innate desire to do useful science. "I [am] pleading for the abolition of the word 'use', and for the freeing of the human spirit," is one of the central sentences in Flexner's essay, which is being republished by Princeton University Press with a companion essay by Robbert Dijkgraaf, Flexner's present successor at Princeton. Dijkgraaf gives some historical background to Flexner's article and demonstrates that his arguments have not lost any of their power and persuasiveness in the modern context of today's research culture.

It is interesting to see that these considerations resonate in parallel with thoughts and reflections found in the philosophy of science. Thomas Kuhn, the most prominent philosophical analyst of scientific revolutions, was one of the first to point out the complexity of scientific progress. He argued that a scientific discovery requires contributions from many individuals, as well as the ability to finally break the dominant paradigm by introducing new perspectives. Such new perspectives seem more likely to emerge if the established, "predictable" paths are abandoned by people "being little committed by prior practice to the traditional rules [...]", as Kuhn puts it.

We, however, currently live in a time when trust in researchers is low and the demand for metric measures of success and thoroughly protocolled short-term goals is high. Astronomy is one of the disciplines that are often publicly accused of not having strong practical relevance, but rather being an activity based almost entirely on human curiosity. Flexner's essay demonstrates how far short of the mark such accusations fall. In times of global scepticism towards science in general and towards fundamental research in particular, and in times when the "freedom of the human spirit" still needs our dedicated protection, Flexner's article is certainly to be as timely a read today as it was 80 years ago.

\section{REVIEWED BY SIBYLLE ANDERL}

Sibylle Anderl is a science editor at the Frankfurter Allgemeine Zeitung and a visiting scientist at the Institut de Planétologie et d'Astrophysique de Grenoble. 